\title{
Creation of a Rapid High-Fidelity Aerodynamics Module for a Multidisciplinary Design Environment
}

\author{
Muktha Srinivasan $^{\mathrm{i}}$, William Whittecari ${ }^{\mathrm{ii}}$, Stephen Edwardsiii, Dimitri N. Mavris ${ }^{\mathrm{iv}}$ \\ Aerospace Systems Design Laboratory, Georgia Institute of Technology, Atlanta, GA 30332
}

\begin{abstract}
$I_{\text {in }}^{\mathrm{n}}$ $\mathrm{n}$ the traditional aerospace vehicle design process, each successive design phase is accompanied by an increment in the modeling fidelity of the disciplinary analyses being performed. This trend follows a corresponding shrinking of the design space as more and more design decisions are locked in. The correlated increase in knowledge about the design and decrease in design freedom occurs partly because increases in modeling fidelity are usually accompanied by significant increases in the computational expense of performing the analyses. When running high fidelity analyses, it is not usually feasible to explore a large number of variations, and so design space exploration is reserved for conceptual design, and higher fidelity analyses are run only once a specific point design has been selected to carry forward.
\end{abstract}

The designs produced by this traditional process have been recognized as being limited by the uncertainty that is present early on due to the use of lower fidelity analyses. For example, uncertainty in aerodynamics predictions produces uncertainty in trajectory optimization, which can impact overall vehicle sizing. This effect can become more significant when trajectories are being shaped by active constraints. For example, if an optimal trajectory is running up against a normal load factor constraint, inaccuracies in the aerodynamic coefficient predictions can cause a feasible trajectory to be considered infeasible, or vice versa. For this reason, a trade must always be performed between the desired fidelity and the resources available.

Apart from this trade between fidelity and computational expense, it is very desirable to use higher fidelity analyses earlier in the design process. A large body of work has been performed to this end, led by efforts in the area of surrogate modeling. In surrogate modeling, an up-front investment is made by running a high fidelity code over a Design of Experiments (DOE); once completed, the DOE data is used to create a surrogate model, which captures the relationships between input variables and responses into regression equations. Depending on the dimensionality of the problem and the fidelity of the code for which a surrogate model is being created, the initial DOE can itself be computationally prohibitive to run. Cokriging, a modeling approach from the field of geostatistics, provides a desirable compromise between computational expense and fidelity. To do this, cokriging leverages a large body of data generated by a low fidelity analysis, combines it with a smaller set of data from a higher fidelity analysis, and creates a kriging surrogate model with prediction fidelity approaching that of the higher fidelity analysis.

When integrated into a multidisciplinary environment, a disciplinary analysis module employing cokriging can raise the analysis fidelity without drastically impacting the expense of design iterations. This is demonstrated through the creation of an aerodynamics analysis module in NASA's OpenMDAO framework. Aerodynamic analyses including Missile DATCOM, APAS, and USM3D are leveraged to create high fidelity aerodynamics decks for parametric vehicle geometries, which are created in NASA's Vehicle Sketch Pad (VSP). Several trade studies are performed to examine the achieved level of model fidelity, and the overall impact to vehicle design is quantified.

1. Hampsten, K. "Spacelift Development Plan."

\section{References}

www.acq.osd.mil/nsso/conference/briefs/HampstenSDP\%20Public\%20Release.ppt

2. Hampsten, K. and Hickman, R. A., "Next Generation Air Force Spacelift," AIAA 2010-8723, AIAA SPACE 2010 Conference \& Exposition, Anaheim, California, August 30 - September 2, 2010.

3. Griffin, K, and Pendleton, E.," A Hybrid Launch Vehicle Design Concept Based on Recent Industry Studies, A Consensus View," Paper AIAA 2008-1135, Proceedings of the 46th AIAA Aerospace Sciences Meeting, Reno, Nevada, January 7-10, 2008.

\footnotetext{
${ }^{\text {i }}$ Graduate Research Assistant, School of Aerospace Engineering, 270 Ferst Dr, Mail Stop 0150, AIAA Member.

${ }^{i i}$ Graduate Research Assistant, School of Aerospace Engineering, 270 Ferst Dr, Mail Stop 0150, AIAA Member.

iii Research Engineer, School of Aerospace Engineering, 270 Ferst Dr, Mail Stop 0150, AIAA Member.

${ }^{\text {iv }}$ Boeing Professor of Advanced Aerospace Systems Analysis, School of Aerospace Engineering, 270 Ferst Dr, Mail Stop 0150, AIAA Fellow.
} 
4. Biggs, R., Love, M., Pendleton, E., “An Integrated Airframe Experiment for Future Responsive Access to Space Applications.” AIAA-2009-2630, 50th AIAA/ASME/ASCE/AHS/ASC Structures, Structural Dynamics, and Materials Conference, Palm Springs, California, May 4-7, 2009.

5. Reusable Booster Integrated Demo-Concept Options Maturation Study Request for Information. Solicitation Number: RFI-PKV-09-01.

6. Reusable Booster System (RBS) Pathfinder. Solicitation Number: BAA-10-04-PKV.

7. Edwards, S. J., Mavris, D., Hellman, B., "Rocketback Trajectory Figures of Merit for a Reusable Booster Technology Demonstrator," AIAA 2010-8667, AIAA SPACE 2010 Conference \& Exposition, Anaheim, California, August 30 - September 2, 2010.

8. Bradford, J., and St. Germain, B., "Rocketback Trajectory Sensitivity Analyses for a Reusable Booster System," AIAA 2010-8672, AIAA SPACE 2010 Conference \& Exposition, Anaheim, California, August 30 - September 2, 2010. 